\title{
Use of Functional Ambulation Performance Score as measurement of gait ability: Review
}

\author{
Arnaud Gouelle, PhD \\ Gait and Motion Analysis Unit, Hôpital Robert Debré, Paris, France
}

\begin{abstract}
Gait analysis systems are widely used for the assessment of gait disabilities and provide more accurate and detailed information than clinical tests. Scores and indexes have been proposed to summarize the large volume of data produced, each emphasizing different aspects of gait. Based on specific spatiotemporal gait parameters, the Functional Ambulation Performance Score (FAPS) quantifies gait at a selfselected speed. Integrated within electronic walkways, the FAPS is commonly used for clinical evaluations and has been used in an increasing number of publications over the past few years. However, its use is sometimes distorted by misunderstandings of its composition and calculation, practical and/or conceptual limits, and even the meaning of the score. This technical report reviews the use of the FAPS for the evaluation of gait based on peer-reviewed articles and clinical experience and addresses important issues that must be considered for an optimal unbiased understanding and analysis of the score.
\end{abstract}

Key words: assessment, elderly, functional ambulation performance, functional ambulation profile, gait, multiple sclerosis, Parkinson disease, score, spatiotemporal gait parameters, walking speed.

\section{INTRODUCTION}

Spatiotemporal gait parameters (STPs) are widely used to evaluate gait abnormalities in patients with neurological and orthopedic disorders as well as in elderly people. Current electronic walkway systems automati- cally collect this valuable clinical information. In order to integrate data and provide a single numerical representation of gait, different scores and indexes have been developed, each emphasizing different aspects. For example, a conglomerate measure based on nine STPs called the Gait Variability Index has been proposed recently to quantify gait variability [1]. An older measure, the Functional Ambulation Performance Score (FAPS) focuses on functional aspects of gait and represents a quantification of patients' gait based on a selection of mean STPs obtained at a self-selected speed [2]. The FAPS is integrated within the GAITRite walkway (CIR System Inc; Clifton, New Jersey), which is considered to be the gold standard for STP analysis. The FAPS is commonly used for clinical evaluations [3], and the number of publications in which it is used has been increasing since 2009 .

However, use of the FAPS is sometimes distorted by misunderstandings of its composition and calculation, practical and/or conceptual limits, and even the meaning of the score. Indeed, some studies have used the FAPS to

Abbreviations: FAPS $=$ Functional Ambulation Performance Score, $\mathrm{ICC}=$ intraclass coefficient, $\mathrm{LL}=$ leg length, $\mathrm{SL}=$ step length, STP $=$ spatiotemporal gait parameter, $\mathrm{V} / \mathrm{LL}=$ normalized velocity.

Address all correspondence to Arnaud Gouelle, PhD; APHP, Hôpital Robert Debré, Plateforme d'Analyse du Mouvement, 48 boulevard Sérurier, 75019 Paris, France; +0033-(0)6-99-02-00-39. Email: arnaud.gouelle@gmail.com http://dx.doi.org/10.1682/JRRD.2013.09.0198 
quantify gait variability [4], and the score has been used as an indication of stride to stride variability [5] despite the fact that the FAPS does not quantify variability. This is therefore a problem when the FAPS is used as an outcome measure for a protocol [6], as a "ground truth for fall risk assessment" [7], or to determine the concurrent validity of a clinical gait and balance scale [8]. It seems that there is a need for some clarification about the history of the score, what it measures, calculation, validity, and possible limitations.

This technical report reviews the use of the FAPS for the evaluation of gait based on peer-reviewed articles and clinical experience and addresses important issues and recommendations regarding clinical use and experimental design.

\section{METHODS}

Two searches were carried out in Science Direct and PubMed using the following subject heading terms: (1) "functional ambulation" AND "profile" OR "performance" OR "score," and (2) "GAITRite" AND "score." No limitations or time period restrictions were applied and the latest search was carried out online on August 31, 2013. All studies in which the FAPS was used were included, regardless of the type of article (e.g., prospective and retrospective studies, published conference abstracts, case reports). Then, attempts to identify further articles were made by searching in the references of the studies found.

\section{RESULTS}

\section{History}

Many authors who use the FAPS cite work by Arthur J. Nelson (1974) [9]. This author discusses the original idea that underlies the FAPS: to create an objective measure of gait capacity. He does not define a score, but rather a sequence of clinical tests that he calls the Functional Ambulation Profile. This first description of the Functional Ambulation Profile consisted of a three-part paper-and-pencil test based on the principle that walking ability depends on subordinate skills that can be identified and measured. In this way, each part of the test served to estimate a subskill of walking: the capacity to (a) create a stable base of support in an orthostatic posi- tion, on both legs and on each leg separately, with and without support from the hand; (b) transfer body weight from one limb to the other in a rhythmical and rapid manner; and (c) alternately transfer body weight when moving forward. For each test, the clinician marked on a grid if it was achieved, the length of time achieved, and the number of steps for the gait tests.

The first reference to use of such a numeric score was in 1986. Glasser used a computerized Functional Ambulation Profile system, designed and developed by Tucker and Nelson, but that was not described in detail [10]. According to her study carried out in hemiparetic patients [10], it would appear that the score was calculated from STPs. A higher score showed an improvement in overall ambulation performance. However, little information is available regarding the method of calculation and thus it is difficult to understand exactly what the score represented.

It was not until 1995 that the current form of the score was formalized. Arthur J. Nelson and Constantin Trantzas worked together to develop a system that could automatically record the necessary parameters and calculate the score: this was how the FAPS and the GAITRite electronic walkway were created.

The article by Gretz et al. in 1998, on the subject of the gait of adults with Down syndrome, was the first publication to present results using the FAPS [2].

Because of this history and the name used in the GAITRite software (i.e., Functional Ambulation Profile), the terminology "Functional Ambulation Profile" is often inappropriately used in place of "Functional Ambulation Performance Score," although they refer to different tests. The inexact term "Functional Ambulatory Profile" can even be found in the literature (Table).

\section{Calculation}

The FAPS is calculated by subtracting points from a maximum score of 100 for a self-selected velocity gait trial (Figure 1). The following parameters are needed: step time (in seconds), step length (SL) to leg length (LL) ratio, normalized velocity (V/LL) for each leg, degree of asymmetry for SL/LL ratio between both limbs, and the dynamic base of support (i.e., the distance from the center of the heel of one footprint to the line of progression formed by two footprints of the opposite foot). Lowerlimb length is represented by the distance from the greater trochanter to the floor, transecting the lateral malleolus (if shoes are worn, their thickness must be added). 
Table.

Selected information from peer-reviewed articles using Functional Ambulation Performance Score (FAPS).

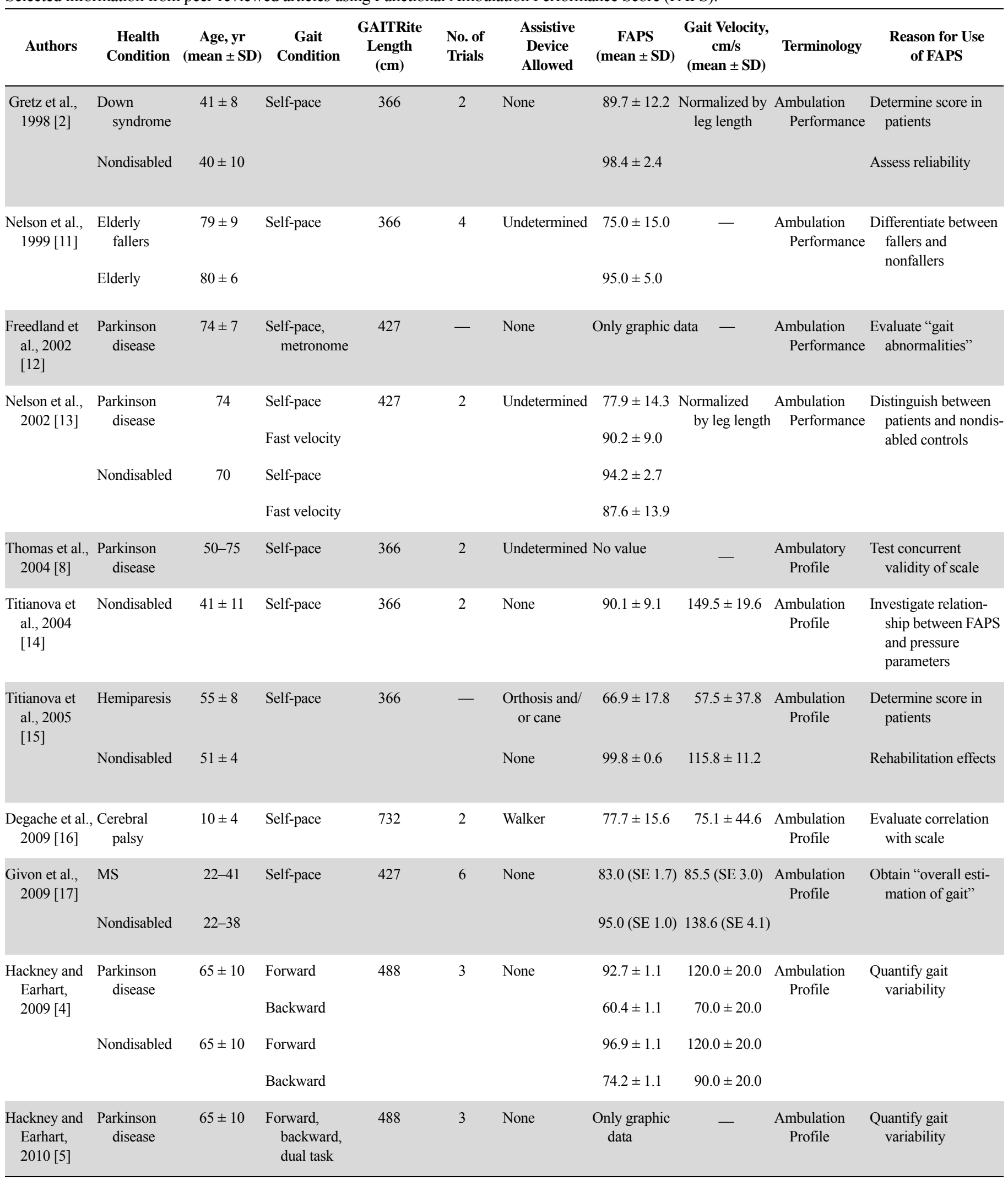


JRRD, Volume 51, Number 5, 2014

Table. (cont)

Selected information from peer-reviewed articles using Functional Ambulation Performance Score (FAPS).

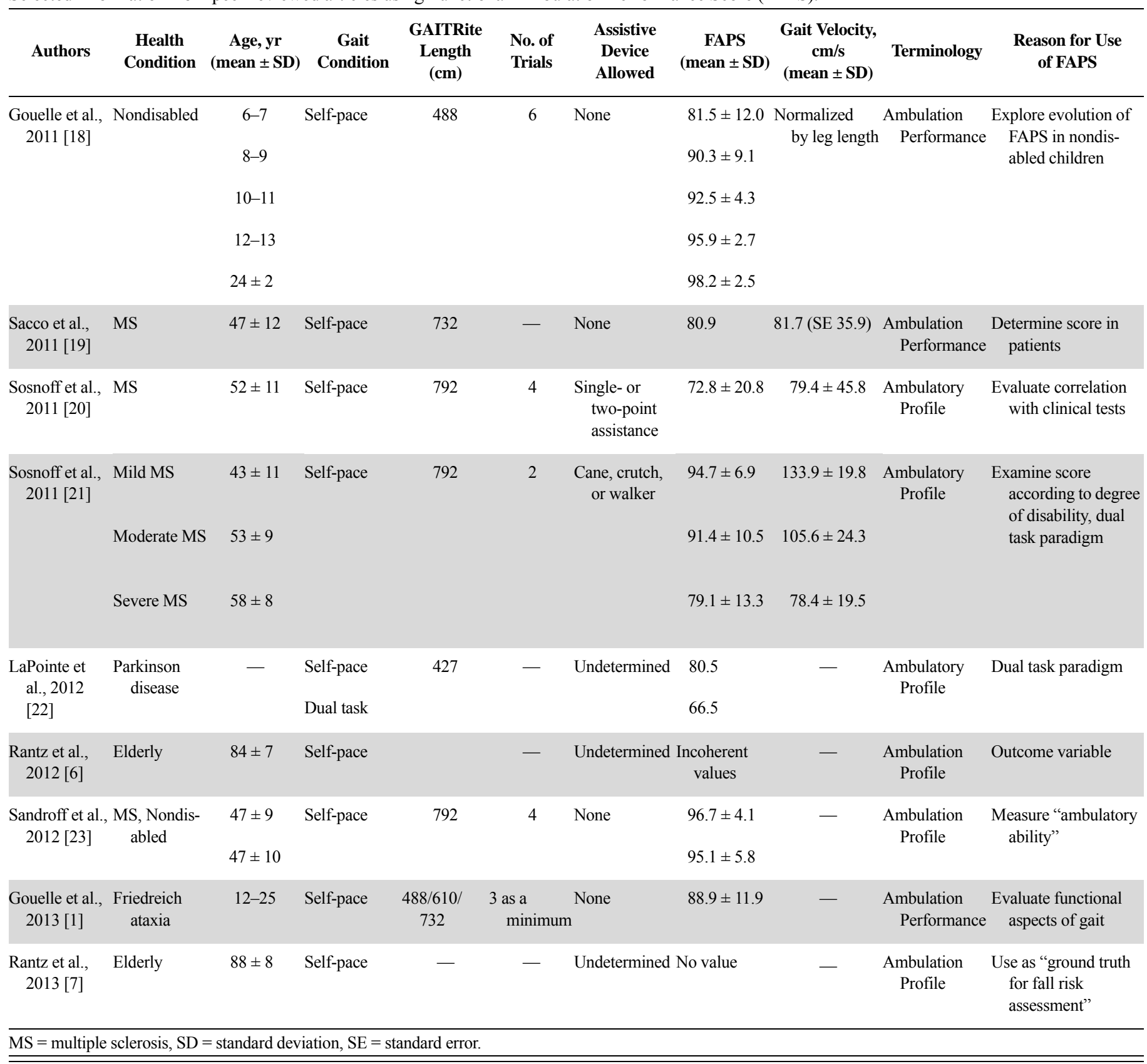

The score calculated for each parameter is then used in four different categories [18]:

- Left and right step functions: The normal values of the $\mathrm{SL} / \mathrm{LL}$ ratio, the step time, and the V/LL ratio are plotted on a double abscissa chart that delimits a zone of "normal values" (Figure 2). The farther the patient's values are from this area, the greater the number of points to deduct (from 0 to 22 points for each side [left and right]).

- Differential of SL/LL ratio: The absolute difference between left and right SL/LL is used to calculate the differential. Up to 8 points are deducted from the right-left asymmetry of the SL/LL ratio if this value is above a normal range. 


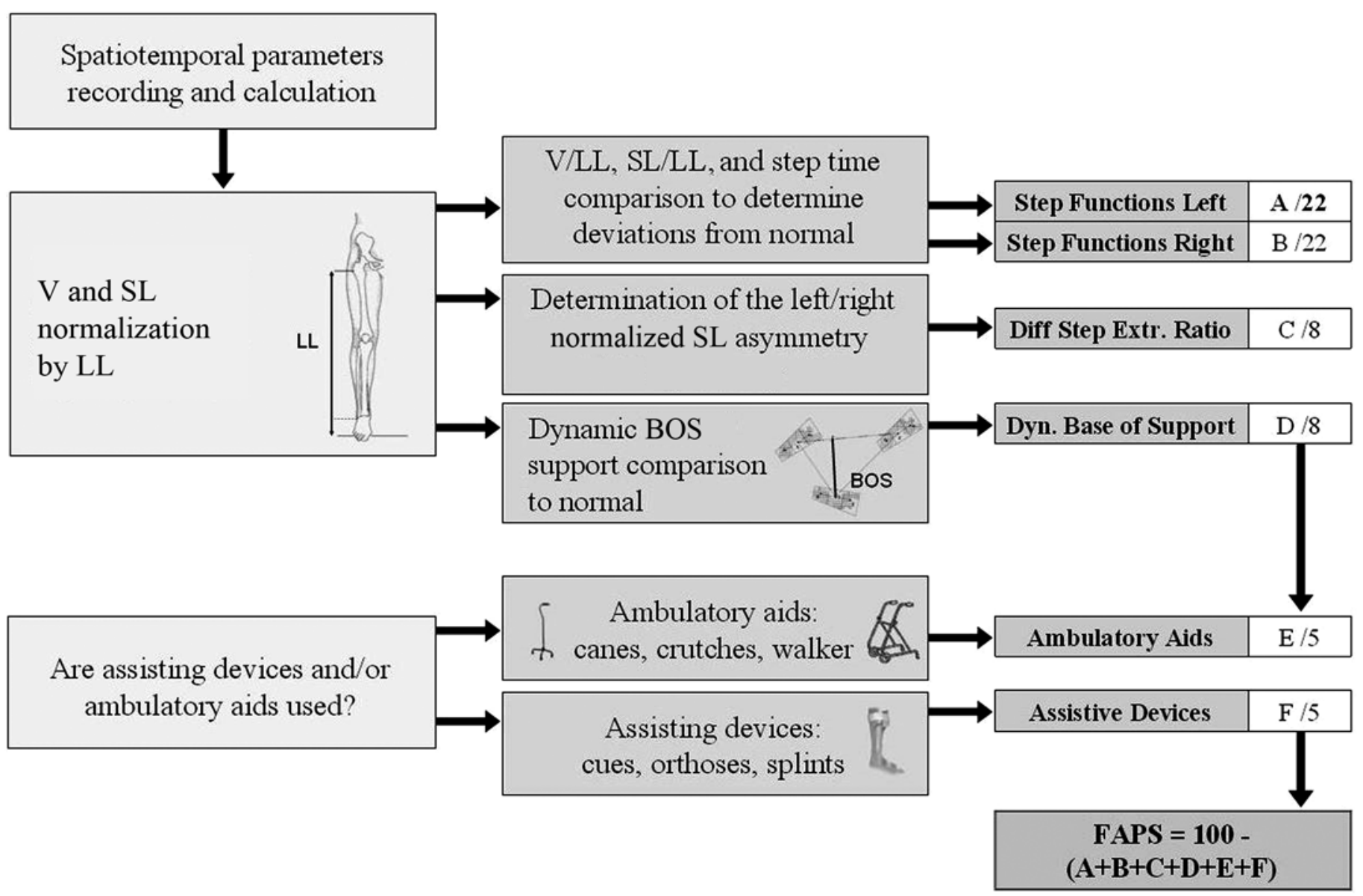

Figure 1.

Flowchart of Functional Ambulation Performance Score (FAPS) calculation. BOS $=$ base of support, Diff $=$ differential, Dyn $=$ dynamic, Extr = extremities, $\mathrm{LL}=$ leg length, $\mathrm{SL}=$ step length, $\mathrm{V}=$ velocity.

- Dynamic base of support: Up to 8 points are deducted if the base of support is abnormally wide or narrow (i.e., if the patient crosses his/her feet).

- Ambulatory aids and assistive devices: Further points can be deducted if walking aids (e.g., canes, crutches, or walkers) (5 points) or assistive devices (e.g., verbal cues, orthoses, or splints) (5 points) are used.

If points are deducted for the use of walking aids or assistive devices, as defined in the software, the lowest possible score is 30 , with a range from 30 to 100 . If walking aids and devices are not taken in to account, the lowest possible score is 40 (range 40-100). In the nondisabled adult population, FAPS ranges from 95 to 100 points (Table) [18].

\section{Validity and Reliability}

High levels of concurrent validity and test-retest reliability have been found for the GAITRite system in different populations [24-25]. For V/LL, normalized SL, step time, and base of support, overall intraclass coefficients (ICCs) above 0.88 were found in a group of nondisabled adults and in a group of adults with Down syndrome [2]. In these groups, ICC values for FAPS were 0.90 and 0.92 , respectively [2]. Sosnoff et al. found an ICC of 0.99 for the FAPS over four consecutive walking trials in persons with multiple sclerosis [20].

Studies that used the FAPS to evaluate pathological gait have shown that the test can be used to distinguish between patients and nondisabled subjects, with lower scores indicating poorer gait (Table). This has been 


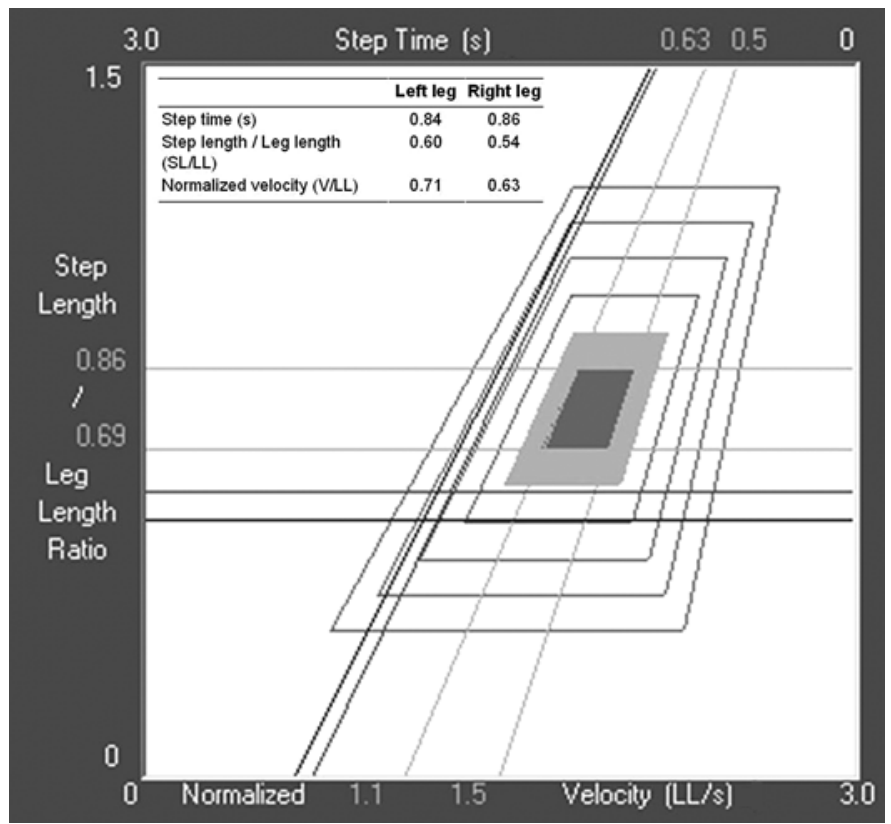

Figure 2.

Details of graphic-based calculation for deductions in left and right step functions categories. Predetermined values of step length (SL)/leg length (LL) ratio (from 0.69-0.86), step time (from 0.5 to $0.63 \mathrm{~s}$ ), and normalized velocity/LL ratio (1.1-1.5 LL/s) are plotted on double abscissa chart to delimit areas that determine number of points to deduct. Patient's parameters (example data given in Table) are then plotted for right and left legs and compared with normal range: dark shaded area in center represents area for which no points are removed for "Step Functions" categories.

found for persons with Down syndrome [2], Parkinson disease [4,12,22], hemiparesis [15], multiple sclerosis [17,19-21,23], Friedreich ataxia [1], and elderly fallers [11]. Use of a dual task paradigm [21-22] and backward walking [4-5] also reduces the score.

The results of the FAPS have also been correlated with different tests and clinical scales. A strong negative association has been found between the FAPS and the level of neurological disability in patients with multiple sclerosis assessed by the Expanded Disability Status Scale with a Pearson correlation coefficient of -0.85 ( $p<$ $0.001)$ [19] and $-0.72(p<0.001)$ [17]; a lower FAPS indicated higher levels of impairment. Negative correlations were also found between the FAPS and walking performance tests with a lower FAPS for longer times to achieve the test. Timed Up and Go test and FAPS were correlated with a Pearson coefficient of $-0.80(p<0.001)$ in an elderly sample [7] and a Spearman rho $(\rho)$ of -0.88 $(p<0.05)$ in a sample with multiple sclerosis [20]. Comparable results were found between the FAPS and the timed $25 \mathrm{ft}$ walk test $(\rho=-0.82, p<0.05)[20]$. A strong positive association was found between the FAPS and a self-reported measure of functional limitations and disability (Late Life Function and Disability Inventory) ( $\rho=$ $0.81, p<0.05$ ) [20], with higher scores indicating higher levels of functioning. These results suggest that the FAPS is a valid marker of gait impairment, particularly in multiple sclerosis.

\section{DISCUSSION}

Several important aspects of the FAPS must be kept in mind when using the test for clinical evaluation or research because they could constitute limitations to the test. This relates to both practical aspects (recording and calculation) and the design of the FAPS itself.

The first point concerns taking into account devices and gait aids. As previously described, 10 further points can be deducted for the use of devices. However, five points are systematically deducted no matter what the type of aid, e.g., simple verbal cues, one single cane, two quad canes, a walker, or parallel bars (see the complete list in the Codes tab in Profile Module of the GAITRite software). For example, two patients with similar gait parameters, but one using a walking frame and the other simple verbal cues, will have the same FAPS score. The number of points can be determined by the user: for example, two points could be subtracted for the use of a stick and five for a walking frame. Personalization of the deduction, however, poses a problem for the comparisons of scores between different articles using the FAPS. It would therefore seem more appropriate not to take aids/ devices into account in the calculation of the FAPS. Another solution could be to specify the FAPS followed by the number of points attributed to gait aids (e.g., a score of 65(10)), as is the case in the PKMAS software (ProtoKinetics; Havertown, Pennsylvania).

Users of the GAITRite software must be aware of the way in which the system deals with recorded trials. One trial on the electronic mat creates a "Walk" file, and several trials can be grouped in a "Test" file. When this is done, the software assimilates the different trials as a single global recording and calculates velocity by dividing the total distance walked by the total gait time. As such, 
when trials are put together in a "Test" file, relatively different gait patterns (e.g., gait that varies from trial to trial or fatigue during the last trial) or velocities are pooled and therefore the resulting FAPS calculated will not represent the patient's true gait. For this reason, we believe that it is preferable to calculate the FAPS for each trial and then to calculate the mean and the dispersion using standard deviations.

Several authors have noted problems related to the design of the FAPS. A critical problem is the use of normative databases that are not adapted to all subjects or protocols. Another problem is the level of importance given to gait velocity in the score. The number of points deducted in the different parts of the FAPS is determined by the distance between the subject's parameters and ranges of predefined values considered as "normal" for gait at a spontaneous velocity. Therefore, in order for no points to be deducted for the "left step functions" and "right step functions" that are visually scored using the position plotted in the central, dark gray area (Figure 2), gait V/LL should be 1.1 to $1.5 \mathrm{LL} / \mathrm{s}$, with a step time of 0.5 to $0.63 \mathrm{~s}$ and a normalized SL/LL of 0.69 to 0.86 . It means that the distance covered each second by a nondisabled subject walking at self-selected speed should be between 1.1 and 1.5 times the LL and that the SL should represent between 69 and 86 percent of the LL. For gait slower than $1.1 \mathrm{LL} / \mathrm{s}$ or faster than $1.5 \mathrm{LL} / \mathrm{s}$, the number of points subtracted increases. What are the practical consequences of this? Some protocols have been designed to evaluate changes in STPs and FAPS during fast gait in patients and nondisabled subjects $[13,15]$. During gait at a comfortable velocity, nondisabled subjects' FAPS is near 100, while for patients, who frequently have a slower gait, the score is reduced. For fast gait, the patient's velocity tends to move toward the "norm" while that of nondisabled subjects moves away from it; the patients' scores thus improve and the nondisabled subjects' scores worsen. The figure shown in the article by Titianova et al. showing FAPS for stroke patients and nondisabled subjects with gait speeds from 20 to $230 \mathrm{~cm} / \mathrm{s}$ is a good example [15]. This explains why Nelson et al. found no significant difference between the FAPS of patients with Parkinson disease $(90 \pm 9)$ and nondisabled subjects ( $88 \pm$ 14) during fast gait [13]. This specificity must be taken into account if the FAPS is used clinically, for example, to evaluate the effects of a rehabilitation program. For the same reason, the FAPS cannot be used to evaluate children under the age of $12 \mathrm{yr}$ [18].
The FAPS is strongly dependent on velocity, step time, and SL (with up to 44 points deducted from the final score). Base of support and asymmetrical SL only make up a maximum of 16 points. This explains the strong correlation between the FAPS and the Timed Up and Go Test [7] and the timed $25 \mathrm{ft}$ walk [20] but leads to question if using the FAPS has any advantage over simply measuring gait velocity [20]. Lastly, the design of the FAPS can also result in a floor effect because the maximum deduction of 22 points each for left and right functions yields a score of 56. As Gretz et al. write, "at this level, the FAPS sensitivity to the amount of temporal distance gait measure differences from the normal ranges is low" [2].

\section{CONCLUSIONS}

It is logical that with the rapid increase in the number of systems for the recording of STP and the integration of the FAPS within some of them, this outcome measure should be used and published in the literature. The primary aim of the FAPS - to objectively evaluate changes in gait related to the pathology and to provide a tool that takes more factors into account than simply gait velocity, for example - is clinically indisputable. Although several studies have shown the FAPS's validity and clinical usefulness, it is important to be aware of some of the characteristics of the FAPS in order to carry out an informed analysis of the results, particularly that the method of calculation limits its use, especially for higher gait velocities.

\section{ACKNOWLEDGMENTS}

Financial Disclosures: The author has declared that no competing interests exist.

Funding/Support: This work was unfunded at the time of manuscript publication.

\section{REFERENCES}

1. Gouelle A, Mégrot F, Presedo A, Husson I, Yelnik A, Penneçot GF. The gait variability index: A new way to quantify fluctuation magnitude of spatiotemporal parameters during gait. Gait Posture. 2013;38(3):461-65. [PMID:23889886] http://dx.doi.org/10.1016/j.gaitpost.2013.01.013 
2. Gretz HR, Doering LL, Quinn J, Raftopoulos M, Nelson AJ, Zwick DE. Functional ambulation performance testing of adults with Down syndrome. NeuroRehabilitation. 1998;11(3):211-25. [PMID:24525924] http://dx.doi.org/10.1016/S1053-8135(98)00023-7

3. Lucet A, Mégrot F, Gouraud D. [Integration of gait analysis tools in the therapeutic approach during the longitudinal therapeutic follow-up of $\mathrm{CP}$ patients]. J Réadaptat Med. 2010;30(3):115-23. French. http://dx.doi.org/10.1016/j.jrm.2010.05.003.

4. Hackney ME, Earhart GM. Backward walking in Parkinson's disease. Mov Disord. 2009;24(2):218-23. [PMID:18951535] http://dx.doi.org/10.1002/mds.22330

5. Hackney M, Earhart G. The effects of a secondary task on forward and backward walking in Parkinson Disease. Neurorehabil Neural Repair. 2010;24(1):97-106. http://dx.doi.org/10.1177/1545968309341061

6. Rantz MJ, Skubic M, Koopman RJ, Alexander GL, Phillips L, Musterman K, Back J, Aud MA, Galambos C, Guevara RD, Miller SJ. Automated technology to speed recognition of signs of illness in older adults. J Gerontol Nurs. 2012; 38(4):18-23. [PMID:22420519] http://dx.doi.org/10.3928/00989134-20120307-01

7. Rantz MJ, Skubic M, Abbott C, Galambos C, Pak Y, Ho DK, Stone EE, Rui L, Back J, Miller SJ. In-home fall risk assessment and detection sensor system. J Gerontol Nurs. 2013;39(7):18-22. [PMID:23675644] http://dx.doi.org/10.3928/00989134-20130503-01

8. Thomas M, Jankovic J, Suteerawattananon M, Wankadia S, Caroline KS, Vuong KD, Protas E. Clinical gait and balance scale (GABS): Validation and utilization. J Neurol Sci. 2004;217(1):89-99. [PMID:14675615] http://dx.doi.org/10.1016/j.jns.2003.09.005

9. Nelson AJ. Functional ambulation profile. Phys Ther. 1974;54(10):1059-65. [PMID:4424703]

10. Glasser L. Effects of isokinetic training on the rate of movement during ambulation in hemiparetic patients. Phys Ther. 1986;66(5):673-76. [PMID:3703931]

11. Nelson AJ, Certo LJ, Lembo LS, Lopez DA, Manfredonia EM, Vanichpong SK, Zwick D. The functional ambulation performance of elderly fallers and non-fallers walking at their preferred velocity. NeuroRehabil. 1999;13(3):141-46.

12. Freedland RL, Festa C, Sealy M, McBean A, Elghazaly P, Capan A, Brozycki L, Nelson AJ, Rothman J. The effects of pulsed auditory stimulation on various gait measurements in persons with Parkinson's Disease. NeuroRehabilitation. 2002;17(1):81-87. [PMID:12016350]

13. Nelson AJ, Zwick D, Brody S, Doran C, Pulver L, Rooz G, Sadownick M, Nelson R, Rothman J. The validity of the GaitRite and the Functional Ambulation Performance scor- ing system in the analysis of Parkinson gait. NeuroRehabilitation. 2002;17(3):255-62. [PMID:12237507]

14. Titianova EB, Mateev PS, Tarkka IM. Footprint analysis of gait using a pressure sensor system. J Electromyogr Kinesiol. 2004;14(2):275-81. [PMID:14962780]

http://dx.doi.org/10.1016/S1050-6411(03)00077-4

15. Titianova EB, Mateev PS, Peurala SH, Sivenius J, Tarkka IM. Footprint peak time and functional ambulation profile reflect the potential for hemiparetic gait recovery. Brain Inj. 2005;19(8):623-31. [PMID:16175816]

http://dx.doi.org/10.1080/02699050400013634

16. Degache F, Perrier M, Bayle B, D'Anjou MC, Gautheron V. [Study of relationships between the Gillette scale and walking speed in children with cerebral palsy]. Motricité Cérébrale. 2009;30(3):97-102. French.

http://dx.doi.org/10.1016/j.motcer.2009.06.002

17. Givon U, Zeilig G, Achiron A. Gait analysis in multiple sclerosis: Characterization of temporal-spatial parameters using GAITRite functional ambulation system. Gait Posture. 2009;29(1):138-42. [PMID:18951800] http://dx.doi.org/10.1016/j.gaitpost.2008.07.011

18. Gouelle A, Mégrot F, Presedo A, Penneçot GF, Yelnik A. Validity of Functional Ambulation Performance Score for the evaluation of spatiotemporal parameters of children's gait. J Mot Behav. 2011;43(2):95-100. [PMID:21298587] http://dx.doi.org/10.1080/00222895.2010.538768.

19. Sacco R, Bussman R, Oesch P, Kesselring J, Beer S. Assessment of gait parameters and fatigue in MS patients during inpatient rehabilitation: A pilot trial. J Neurol. 2011; 258(5):889-94. [PMID:21076978]

http://dx.doi.org/10.1007/s00415-010-5821-z

20. Sosnoff JJ, Weikert M, Dlugonski D, Smith DC, Motl RW. Quantifying gait impairment in multiple sclerosis using GAITRite technology. Gait Posture. 2011;34(1):145-47. [PMID:21531562] http://dx.doi.org/10.1016/j.gaitpost.2011.03.020

21. Sosnoff JJ, Boes MK, Sandroff BM, Socie MJ, Pula JH, Motl RW. Walking and thinking in persons with multiple sclerosis who vary in disability. Arch Phys Med Rehabil. 2011;92(12):2028-33. [PMID:22133253] http://dx.doi.org/10.1016/j.apmr.2011.07.004

22. LaPointe LL, Maitland CG, Stierwalt JA. Functional Ambulatory Profile (FAP) gait changes during increased cognitive load in Parkinson disease. Parkinsonism Relat Disord. 2012;18(2):S15. http://dx.doi.org/10.1016/S1353-8020(11)70136-5

23. Sandroff BM, Motl RW, Suh Y. Accelerometer output and its association with energy expenditure in persons with multiple sclerosis. J Rehabil Res Dev. 2012;49(3):467-75. [PMID:22773205] http://dx.doi.org/10.1682/JRRD.2011.03.0063 
24. Bilney B, Morris M, Webster K. Concurrent related validity of the GAITRite walkway system for quantification of the spatial and temporal parameters of gait. Gait Posture. 2003;17(1):68-74. [PMID:12535728] http://dx.doi.org/10.1016/S0966-6362(02)00053-X

25. Webster KE, Wittwer JE, Feller JA. Validity of the GAITRite walkway system for the measurement of averaged and individual step parameters of gait. Gait Posture. 2005;22(4): 317-21. [PMID:16274913] http://dx.doi.org/10.1016/j.gaitpost.2004.10.005

Submitted for publication September 5, 2013. Accepted in revised form December 30, 2013.
This article and any supplementary material should be cited as follows:

Gouelle A. Use of Functional Ambulation Performance Score as measurement of gait ability: Review. J Rehabil Res Dev. 2014;51(5):665-74.

http://dx.doi.org/10.1682/JRRD.2013.09.0198

ResearcherID/ORCID: Arnaud Gouelle, PhD: H-3030-2014 
